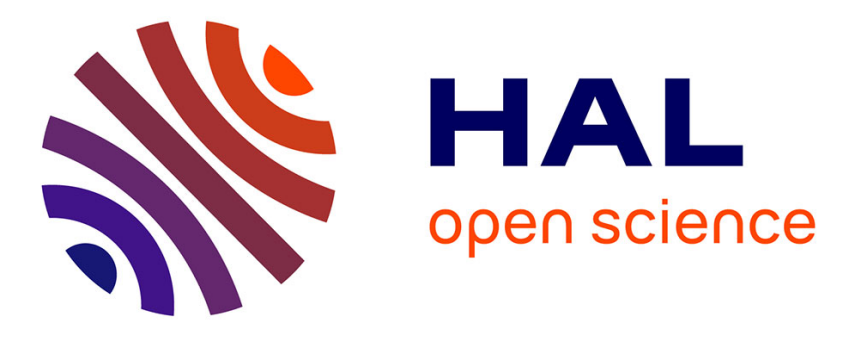

\title{
Statistical Threshold Selection for Path Openings to Detect Cracks \\ Petr Dokládal
}

\section{To cite this version:}

Petr Dokládal. Statistical Threshold Selection for Path Openings to Detect Cracks. International Symposium on Mathematical Morphology and Its Applications to Signal and Image Processing, ISSM 2017, May 2017, Fontainebleau, France. pp.369-380, 10.1007/978-3-319-57240-6_30 . hal-01478089

\section{HAL Id: hal-01478089}

https://hal-mines-paristech.archives-ouvertes.fr/hal-01478089

Submitted on 27 Feb 2017

HAL is a multi-disciplinary open access archive for the deposit and dissemination of scientific research documents, whether they are published or not. The documents may come from teaching and research institutions in France or abroad, or from public or private research centers.
L'archive ouverte pluridisciplinaire HAL, est destinée au dépôt et à la diffusion de documents scientifiques de niveau recherche, publiés ou non, émanant des établissements d'enseignement et de recherche français ou étrangers, des laboratoires publics ou privés. 


\title{
Statistical Threshold Selection for Path Openings to Detect Cracks
}

\author{
Petr Dokládal \\ MINES ParisTech, PSL - Research University, CMM - Centre de morphologie mathématique, 35, rue \\ Saint Honoré, 77300 Fontainebleau, France \\ email: petr.dokladal@mines-paristech.fr
}

\begin{abstract}
Inspired by the a contrario approach this paper proposes a way of setting the threshold when using parsimonious path filters to detect thin curvilinear structures in images.

The a contrario approach, instead of modeling the structures to detect, models the noise to detect structures deviating from the model. In this scope, we assume noise composed of pixels that are independent random variables. Henceforth, cracks that are curvilinear sequences of bright pixels (not necessarily connected) are detected as abnormal sequences of bright pixels.

In the second part, a fast approximation of the solution based on parsimonious path openings is shown.
\end{abstract}

Keywords: Mathematical morphology, parsimonious path filters, rank-max opening, a contrario model

\section{Introduction}

The detection of thin, curvilinear elements is a frequent task in automated visual inspection to detect cracks. Similar applications can be found in other image processing domains to detect the blood vessels (medicine), facial wrinkles (cosmetology), neurites or DNA molecule chain (biology), roads in satellite images, or a few others. An accurate detection of such structures that are thin, curved, discontinuous and often rare and sometimes absent (in which case we want to avoid false detection) from the images is a difficult problem. In mathematical morphology the operator enhancing curvilinear, thin objects is the path opening.

However, the main difficulty to produce a binary results is to find a convenient value for the subsequent thresholding. One solution, based on the same hypothesis formulated using the a contrario model, and combined with the percolation, has been investigated by Aldea and Le Hégarat-Mascle [1]. Long, thin structures are then detected as deviations from normality.

The same hypothesis of sparcity of defects is used throughout this paper. The principal contribution here is the proposition of a method of identifying parameter values such that the detected structures are perceptually significant. The principle is inspired from the idea of meaningful alignments proposed by Desolneux et al. [2], detecting perceptually significant, straight segments in images. The perceptual significance stems from the Helmholtz principle stating that a perceptually significant element is improbable to occur by chance. Any perceptual significant segment is hence a deviation from randomness. The model proposed in [2] is based on the hypothesis of random distribution of the orientation of the isophote ${ }^{1}$ in noise. A perceptually significant segment is defined as a sequence of isophotes aligned with the orientation of the segment. Notice that a perceptually significant segment does not need to be contiguous.

In this work we extend the original model based on the orientation of isophotes to that of the distribution of intensity in images, and from straight segments to curvilinear structures. As well as in the case of the isophotes, the points do not necessarily need to be connected to form a perceptually significant structure, but only sufficiently densely follow each another in a sequence.

\footnotetext{
${ }^{1}$ normal to the gradient direction
} 
This idea can be efficiently implemented using mathematical morphology that indeed possesses an operator conceived for the detection of such structures. It is derived from the original concept of the path opening, due to Buckley and Talbot [3,4], and formalized in Heijmans et al. [5]. Later, Talbot and Appleton [6] propose a more efficient implementation and introduce incomplete path openings robust to noise due to the capability to tolerate missing pixels. The originally exponential complexity has been reduced later to a constant by Morard et al. [7] by limiting the search of paths to only a relevant subset of all paths in an image and using a constant-time opening algorithm for the filtering alongside these paths or a closing-opening to increase the robustnes to noise. Using closing-opening instead of opening alone does not increase the complexity, and is indeed similar to another version proposed by Cokelaer et al. [8] that tolerates gaps up to some maximal admissible width.

The main contribution of the present paper is to set correct parameter values for parsimonious path filters to detect perceptually meaningful structures longer than some chosen minimal length. The morphological tool allowing to implement efficiently the above ideas is parsimonious incomplete path opening applying a 1-D rank-max-opening alongside these paths.

\section{The meaningfulness}

According to the Helmholtz principle, an object is perceived as meaningful provided it is unlikely for it to occur by chance. The complement of these structures is the background that we assume random with a priory unknown distribution.

Definition 1 ( $\varepsilon$-meaningful event (Desolneux et al. [2])). An even of type "such configuration of points has such property" is $\varepsilon$-meaningful if the expectation of the number of occurrences in an image of this event is less than $\varepsilon$.

For an event to be meaningful one needs to consider $\varepsilon \ll 1$, in which case our perception is likely to see it. Notice that the $\varepsilon$-meaningfulness is related to the statistical $p$-significance.

In the following we develop a framework to detect thin, curvilinear structures in gray-scale images. These structures are perceived due to the deviation of their intensity from the normality. They are either brighter or darker. We develop the framework for bright structures; dark structures can be detected after inverting the image. In the simulated experiment Fig. 1 the sinusoidal curve - composed of closely grouped points somewhat brighter than the surrounding - is perceived as one object even though not necessarily contiguous. The difficulty to extract this structure is due to that we do not know the parameters: i) the distribution of the noise, ii) how much the curve points are brighter than the noise, iii) how densely these points populate this curve.

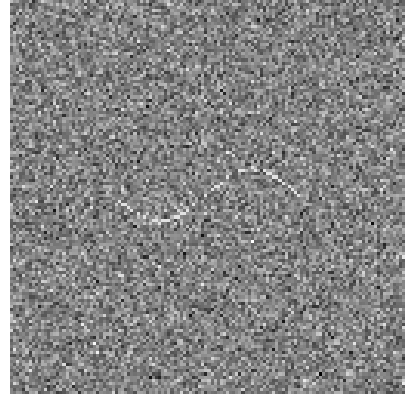

(a)

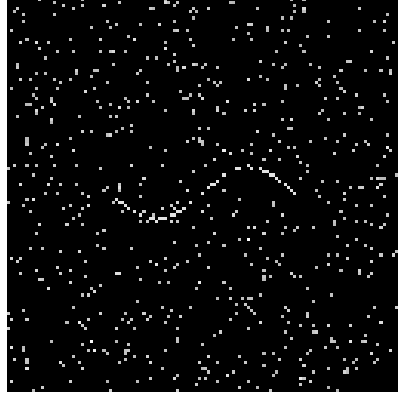

(b)

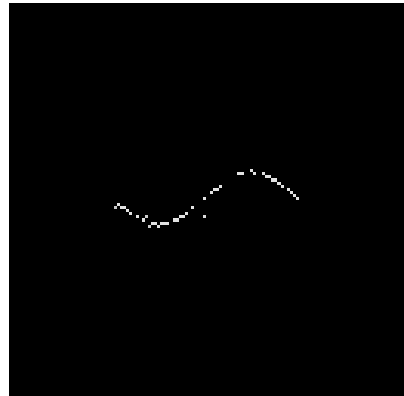

(c)

Fig. 1. a) a curvilinear structure in noise $\mathcal{N}\left(\mu, \sigma^{2}\right)$, b) points above the 88.2 quantile, c) the detected structure with $L=20, k=16$ (see below for parameters setting). 
In the remainder of this text, the ideas from Desolneux et al. [2] originally applied to isophote orientation, are developed and applied to the distribution of gray values. We use identical notation wherever possible and refer the reader for details to [2] for additional details.

In [2] the distribution of orientation of the isophotes in noise is uniformly distributed on $[0,2 \pi]$. It is independent of the values of the pixels, provided the pixels are independent, random variables. The detection of curvilinear structures can not use alignment of isophotes since the local orientation is not constant. We therefore extend the ideas from [2] to the distribution of pixel values in the image. Compared to the distribution of isophotes, the distribution of pixel values not only does not obey the uniform law but is even often unknown. Also an isophote is either aligned or misaligned with the segment. It is a boolean property. Analogously, a "pixel is brighter than" is also boolean but requires some threshold. On the other hand, the statement that "a pixel is bright" does not require a threshold and is not boolean any longer since it can be true to various degrees of truth. Consequently, a curvilinear bright structure will be perceptually meaningful depending of two properties, its brightness and its length. For the same perception stimulus a shorter structure must be brighter than a longer one. Consequently, even a single, isolated pixel in noise can be perceptually meaningful provided it is exceptionally bright. Compared to that, one isophote in noise can never become meaningful.

Let $f$ denote a realization, i.e. a gray-scale image, on a domain $M \times N \subset \mathbb{Z}^{2}$, of a random variable following the law $F$, assumed unknown. The complementary cumulative distribution function

$$
\bar{F}(\alpha)=P\left[f(x)>f_{\alpha}\right]=p
$$

gives the probability $p$ that the value of pixel $x$ exceeds the quantile $\alpha$.

Let $C$ be a curvilinear structure formed by a sequence of $l$ connected $^{2}$ points $\left\{x_{1}, x_{2}, \ldots, x_{l}\right\}$. Let $X_{i}$ be a random variable equal to 1 if $f\left(x_{i}\right)>f_{\alpha}$ and 0 otherwise. Let $S_{l}=X_{1}+X_{2}+\ldots+X_{l}$, with $0 \leq S_{l} \leq l$, be a random variable counting in $C$ the number of points brighter than $f_{\alpha}$. Provided $X_{i}$ are independent we have the probability that exactly $k$ pixels in the curve $C$ exceed the intensity $f_{\alpha}$ given by the binomial distribution

$$
P\left[S_{l}=k\right]=\left(\begin{array}{l}
l \\
k
\end{array}\right) p^{k}(1-p)^{l-k}
$$

\subsection{Number of False alarms}

A path is generated by a connectivity class given by a graph. The vertices of the graph represent the image pixels, and the edges connect pixels that are connected. The graph is directed and acyclic. A path in an image is therefore a path in the graph. The connectivity is assumed translation invariant, except image borders.

Suppose every vertex is connected to $n$ neighbors (with $n=3$ in the example Fig. 6). There are therefore $n^{l-1} l$-pixel-long paths starting in any pixel in $\mathbb{Z}^{2}$. In a $N \times N$ image, the number of possible starting points for a $l$-pixel-long path (generated by either of these graphs) is $(N-l)^{2}$. Hence, the number of $l$-pixel-long paths generated by either of the connectivity graphs in Fig. 6 can be approximated by

$$
\Pi(l ; N)=(N-l)^{2} n^{l-1}
$$

Given the exponential rule we drop the multiplicative factor given by the number of possible rotations of the graph.

Following the idea from [2] we are interested in detecting structures "longer than" rather than "exactly as long as" since the exact length is not known. We set up the detection threshold by decreasing the number of false alarms to below an acceptable (arbitrary but small enough) value.

In statistical terms the presence of a meaningful structure is a test and the number of possible paths along which it can occur is the number of trials. The probability of occurrence multiplied

\footnotetext{
${ }^{2}$ we define connectivity below
} 
by the number of tests gives the expectation of false alarms $N F A$

$$
N F A[k, l]=\Pi(N) P\left[S_{l} \geq k\right]=\sum_{i=k}^{l} \Pi(i, N)\left(\begin{array}{l}
l \\
i
\end{array}\right) p^{i}(1-p)^{l-i}
$$

The upper length limit $l$ in the sum being unknown we let it equal to the length of the diagonal of the image $\sqrt{2} N$. Comparing $N F A$ to $\varepsilon$

$$
N F A[k, l] \leq \varepsilon
$$

is equivalent to saying whether the structure is $\varepsilon$ meaningful.

\subsection{Intensity threshold}

The first thing one can do is to set $k=l$ in eq. 2 and isolate $p$ as a function of the length $l$

$$
p \leq \sqrt[l]{\frac{\varepsilon}{\Pi(l) e^{l-1}}}
$$

Illustrated in Fig. 2a as a function of the length it shows that shorter structures, to be meaningful, must contain points that are less probable, that is more deviating from the normality. If the law of the intensity distribution in an image is known and invertible eq. 4 can be used to determine the intensity threshold to detect meaningful structures. If the law is unknown or not invertible, a rank-order filter $\xi_{1-p}$ in a sliding window with the rank $1-p$ will select points with intensity above the quantile level $1-p$, that is those with probability less or equal $p$.

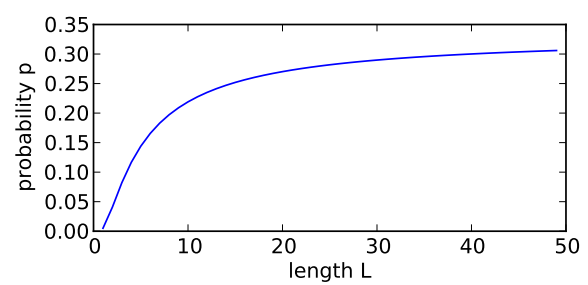

(a)

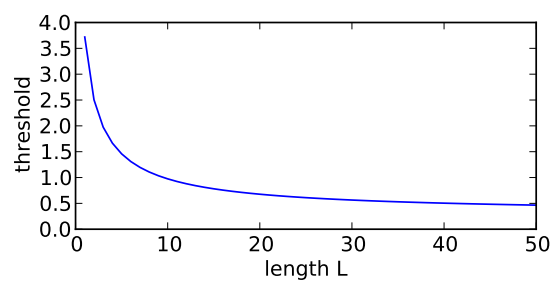

(b)

Fig. 2. a) the probability of point as function of the length, b) the threshold obtained as the quantile level corresponding to $p$ for law $\mathcal{N}\left(\mu, \sigma^{2}\right)$, for $\mu=0, \sigma=1$.

Example: The intensity threshold corresponding to the probability $p$ for a normal law $\mathcal{N}\left(\mu, \sigma^{2}\right)$, with $\mu=0, \sigma=1$, is given by Fig. 2b. It says that even an isolated point becomes meaningful provided its intensity exceeds $3.5 \sigma$. Long, connected structures are meaningful as long as their intensity remains above $0.5 \sigma$ of the noise.

Isolating $k$, with $k \leq l$, from eq. 2 allows saying in how many pixels out of a sequence of $l$ connected pixels the intensity must exceed the $p$-th quantile value for the structure to become meaningful. This means that we search for structures that are composed of bright, not necessarily connected, sequences of points.

$$
k(l)=\min \{k \in \mathbf{N}, N F A[k, l] \leq \varepsilon\}
$$

Given some $l$, eq. 5 can be directly solved for $k$. In a typical image, e.g. $512 \times 512$ pixels, the number of paths $\Pi_{l}$ is a large number, given that it increases exponentially with $l$, itself bounded by the length of the diagonal of the image. 
Consider a collection of $L$ independent, random points. Let $p$ denote the probability that the intensity of a point exceeds some threshold. The probability that in $k$ points out of $L$ the intensity exceeds some fixed threshold follows the binomial law, see illustration Fig. $3 \mathrm{a}$ for $p=0.1$. If these $L$ points form a path in a $\mathbb{Z}^{2}$ grid there are many trials to this test. There are $3^{L-1}$ paths generated by a three-connected graph (either of the graphs in Fig. 6) that start in every point of this grid. This gives a huge number on unbounded (or large) supports, see Fig. $3 \mathrm{~b}$.

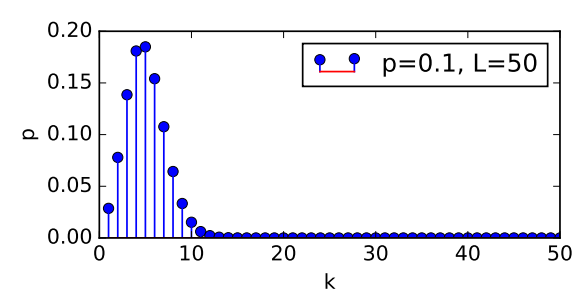

(a)

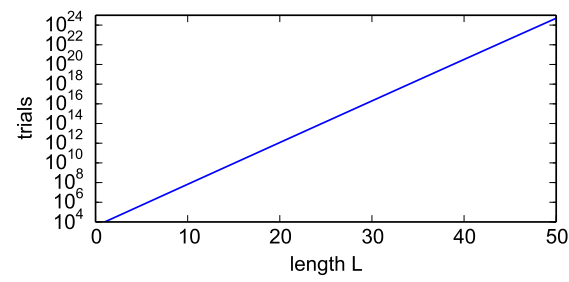

(b)

Fig. 3. (a) Probability mass function of the binomial law, (b) Number of 3-connected paths on $\mathbb{Z}^{2}$ for $L \leq 50$.

The expectation of false alarms $N F A$ is given Fig. 4a. We search for a configuration where $N F A$ in a given image remains low (condition eq. 3) that is the smallest $k$ where the tail of the distribution remains under $\varepsilon$. See Fig. $4 \mathrm{~b}$ for $k$ expressed as a function of $L$, for $p=0.05$.

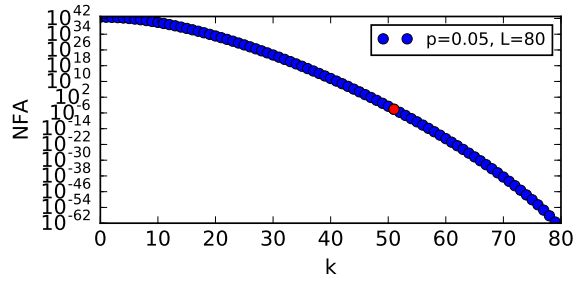

(a)

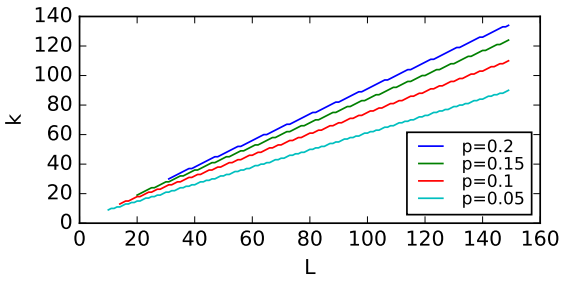

(b)

Fig. 4. (a) NFA as a function of $k$. In red: Choosing $k$ for $\epsilon=0.01$. (b) Minimum $k$ ensuring meaningfulness as a function of the length $L$ and parametrized by $p$.

Solving eq. 3 requires counting the number of paths $\Pi(i, N)$ in eq. 2. Exact counting not being easy on bounded supports, we provide a convenient approximation. Recall that a point in $\mathbb{Z}^{2}$ is an origin for $3^{L-1} L$-pixel-long paths. However, restricting this estimation to bounded supports by multiplying this number by the number of points in a bounded support gives an unacceptably strong overestimation. We propose another, more precise approximation.

A pair of points $(a, b)$ in $\mathbb{Z}^{2}$ is connected by a path which is either i) a line segment running straight from $a$ to $b$ or ii) a path oriented from $a$ to $b$ and containing as many right turns as left turns. The count of such paths is given by

$$
\sum_{k=1}^{L}\left(\begin{array}{l}
L \\
k
\end{array}\right)\left(\begin{array}{c}
L-k \\
k
\end{array}\right)
$$

This number multiplied by the number of possible points $L$ segments distant each from the other. This approximation provides a sufficiently precise estimation of paths on bounded supports. 
Next, we fix some fixed fraction $r=$ const., $r \in \mathbb{R}^{+}, 0<r \leq 1$, for having $k \in \mathcal{N}^{+}$bright pixels in a sequence $k=\left\|r l_{0}\right\|$, with $\|$.$\| meaning rounding to nearest integer. We solve eq. 2$ for the lowest $p$ satisfying the condition on the meaningfulness eq. 3. Observe in Fig. 5 the values of $p$ that we obtain for various $r$. Notice that the curve for $r=1$ coincides with the one given by Fig. 2a (that of a complete path). Observe that for longer curves and higher $r$ lower threshold suffices to ensure meaningfulness. These probabilities allow obtaining correct threshold values for the rank filter $\xi_{r}$, as explained below.

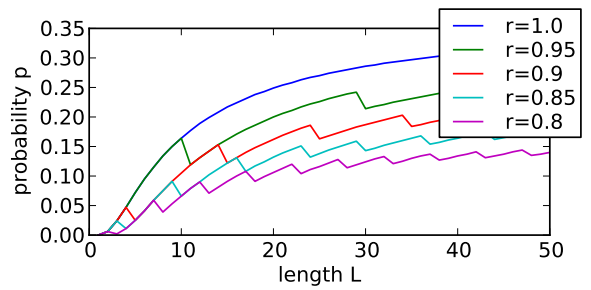

Fig. 5. The pixel probability $p$ as a function of the sequence length $L$ for various fixed fill fraction levels $r$.

\section{Efficient Implementation with morphological tools}

We have seen that an obvious obstacle in the search of efficient implementation is the overwhelming number of trials to test in an image. The test "intensity in $k$ out of $l$ points in a path exceeds a value" done alongside all paths is computationally very intensive; the number of paths in an image increases linearly with the image size and exponentially with the length of the paths. Testing even a much smaller number of trials in the case of straight lines in the algorithm in [2], takes seconds on a recent computer [9]. In what follows we present a convenient fast approximation of the solution using path openings.

Given some function $f: D \rightarrow V$, the morphological opening of $f$ by a structuring element $B \subset D$ defined by

$$
\gamma_{B} f=\sup \{B(u)+v \leq f ;(u, v) \in D \times V\}
$$

is a supremum of all translates of $B$ that do not exceed $f$, where $B(u)+v$ denotes $B$ translated horizontally by $u$ and vertically by $v$. That is, for some $x \in D$, there is a translate $B(u)$ such that for any $y \in B(u), f(y) \geq \gamma_{B} f(x)$. Defining $g=\left\{x \mid \gamma_{B} f(x)>t h\right\}$, then $x \in g$ means that there is some $B(u), x \in B(u)$ that $f(y)>t h$, for all $y \in B(u)$. For a connected $B$ with $|B|=k, g$ indicates where $f>t h$ larger than $k$. In 1-D these subdomains are intervals.

In presence of noise the strict inclusion of $B$ is likely to fail. This motivated the introduction of the rank-max opening, proposed by Ronse [10] and later described in Ronse and Heijmans [11], defined as the supremum of openings by all subsets of cardinal $k$ of a chosen structuring element $B$

$$
\gamma_{B, k}=\bigvee_{i}\left\{\gamma_{B_{i}} \mid B_{i} \subseteq B, \operatorname{card}\left(B_{i}\right)=k\right\}
$$

where $1 \leq k \leq n$ with $n=\operatorname{card}(B)$. It acts as an opening tolerating $n-k$ missing pixels.

Note that $r$, in $r=k / l$, is to be understood as the rate of pixels that are required to be intensive, and inversely $1-r$ the rate of tolerated missing pixels.

A naive implementation of eq. 7 requires computing $\left(\begin{array}{l}n \\ k\end{array}\right)$ openings which is prohibitive for most applications. Instead of the naive implementation, Heijmans [12] shows that eq. 7 can be efficiently 
implemented by using this identity

$$
\gamma_{B, k}=\mathbb{1} \wedge\left(\delta_{\check{B}} \xi_{B, n-k+1}\right)
$$

where $\delta_{\breve{B}}$ is the conjugate dilation with $\check{B}(x)=B(-x)$ and $\xi_{B, r}$ a rank order filter

$$
\xi_{B, r} f(x)=\text { r-th largest value of }\{f(y), y \in B(x)\}
$$

Isolating in some original image $f$ perceptually significant sequences of pixels longer than $L$ can be done by thresholding the rank-max opening $\gamma_{L, k}>\xi_{r}$. An approximate solution can be obtained using parsimonious path openings Morard et al. [7] allowing to test only a fraction of all trials. Once the paths are isolated, both the conjugate dilation and the rank filter implemented by definition are reasonably fast on 1-D data. For time-critical applications one can use fast, $\mathcal{O}(1)$ algorithms for both, e.g. [13] for dilation and [14] for the rank order filter. We apply the morphological 1-D rank-max opening alongside the paths with statistically obtained parameters to detect cracks as statistically meaningful structures in images. The following paragraph shortly recalls the essentials.

Let $f: D \rightarrow V$, with e.g. $V=R^{+}$be an image such that $D$ is a rectangular subset of $Z^{2}$. Suppose $D$ equipped with a translation-invariant, acyclic connectivity graph $G: D \rightarrow \mathscr{P}(D)$, with $\mathscr{P}$ denoting the powerset. We say a sequence $\pi=\left(x_{1}, x_{2}, \ldots, x_{n}\right), n \in \mathbb{N}$, of points is a path if $x_{i+1} \in G\left(x_{i}\right)$ for all $1 \leq i \leq n-1$. The path length is $n$. Points $x_{1}$ and $x_{n}$ are its starting and end points. The starting points are on the edges of the image and each path goes to the facing image edge so that the mean intensity is maximized alongside. The set of the paths generated by $G$ in this way is denoted by $\Pi_{G}$. The opening is applied alongside all paths in $\Pi_{G}$. The complement of $\Pi_{G}$ in $\operatorname{spt}(f)$ is set to 0 .

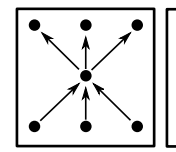

(a)

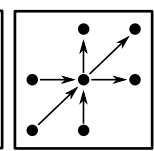

(b)

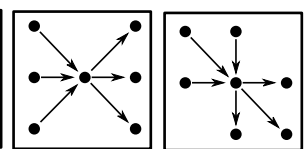

(c)

(d)

Fig. 6. An example of a commonly used connectivity graph (a), and its rotations (b-d)

\section{Results}

We illustrate the method on the detection of road cracks. Prior to the detection of cracks, the original image - where cracks appear dark - is inverted so that an opening can be used to detect the cracks. Notice that even though the background texture is non-stationary no other preprocessing is needed as long as the rank order filter is applied in a sliding window.

This image has a resolution of $1 \mathrm{~mm} /$ pixel in both directions. We choose to detect cracks longer than $L=100 \mathrm{~mm}$. See Fig. 7 for the results.

Notice that discontinuous cracks are still detected but appear as composed of segments that are not necessarily all longer than the parameter $L$. This occurs due to the non-increasingness of the opening (ensured by $\wedge$ in eq. 8 ).

Regarding the timing: the processing time of an $11294 \mathrm{x} 4096$ image is $\sim 8 \mathrm{~s}$ for the rank filter, and $\sim 4$ s the parsimonious rank-max path opening on a $2.70 \mathrm{GHz}$ Intel i7 $\mathrm{CPU}$ (without using the CPU's parallelism capabilities). 

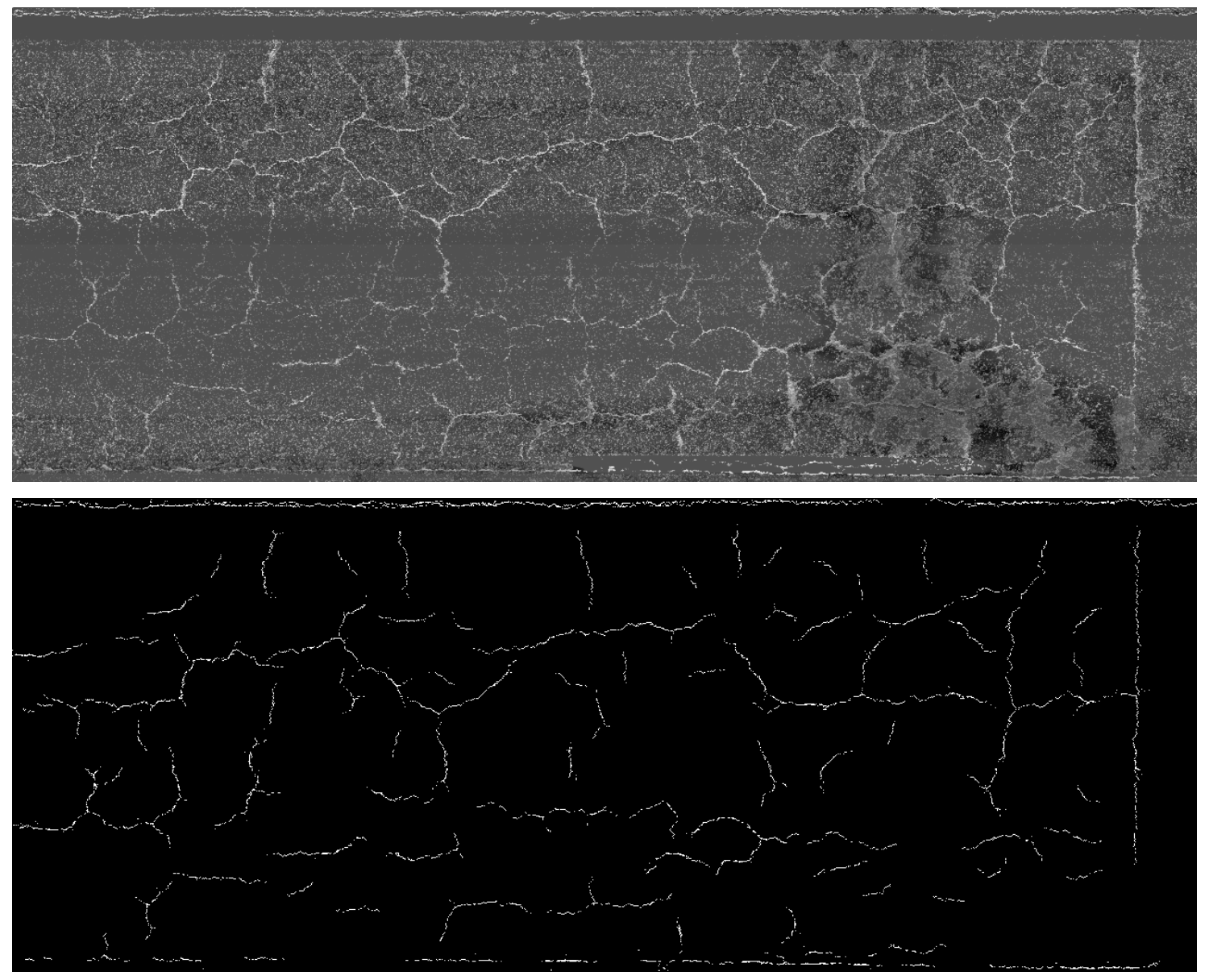

Fig. 7. Detection of road cracks. (top) original, $11294 \times 4096$ image, (bottom) the cracks obtained for $L \geq 100, k=65$ and $r=0.15$. The sliding window is a $100 \times 100$ rectangle.

\section{Properties and discussion}

The result of rank-max path opening is not necessarily composed of connected paths the length of which is equal or greater than $L$ as for path openings. Indeed, the tolerance to noise of rank-max openings makes the result disconnected if the object in the input image is disconnected (as in the simulation Fig. 1 and the road cracks Fig. 7). The rank-max opening may also create small spurious branches due to local random noise arrangements. As a direct consequence, the results obtained with a sequence of increasing lengths are not strictly decreasing (in the sense "being included") though global, non-strict decreasing is observed.

In presence of dense grouping of more cracks the threshold at some quantile is biased upwards which counterbalances the sensitivity. Nonetheless, this comes somewhat in relation with the perception law saying that isolated structures are perceived individually whereas groupings are perceived as a whole first.

One, well known, limitation comes from the path openings. Their underlying acyclic graph puts a limit on the tortuosity of the structures. The length of excessively tortuous structures becomes underestimated. This drawback is however widely accepted since counter-balanced by the linear computational complexity of this algorithm and rapidity even on large images. 


\section{References}

1. E. Aldea and S. Le Hégarat-Mascle, "Robust crack detection for unmanned aerial vehicles inspection in an a-contrario decision framework," Journal of Electronic Imaging, vol. 24, no. 6, pp. 061 119-061 119, 2015.

2. A. Desolneux, L. Moisan, and J.-M. Morel, "Meaningful alignments," International Journal of Computer Vision, vol. 40, no. 1, pp. 7-23, 2000.

3. M. Buckley and H. Talbot, "Flexible linear openings and closings," Computational Imaging and Vision, vol. 18, pp. 109-118, 2000.

4. H. Heijmans, M. Buckley, and H. Talbot, "Path openings and closings," Probability, Networks and Algorithms, no. E 0403, pp. 1-21, 2004.

5. _ _ "Path openings and closings," Journal of Mathematical Imaging and Vision, vol. 22, no. 2, pp. 107-119, 2005.

6. H. Talbot and B. Appleton, "Efficient complete and incomplete path openings and closings," Image and Vision Computing, vol. 25, no. 4, pp. 416-425, 2007.

7. V. Morard, P. Dokládal, and E. Decencière, "Parsimonious path openings and closings," IEEE Transactions on Image Processing, vol. 23, no. 4, pp. 1543-1555, 2014.

8. F. Cokelaer, H. Talbot, and J. Chanussot, "Efficient Robust d-Dimensional Path Operators," Selected Topics in Signal Processing, IEEE journal of, vol. 6, no. 7, November 2012.

9. R. Grompone von Gioi, J. Jakubowicz, J.-M. Morel, and G. Randall, "LSD: a Line Segment Detector," Image Processing On Line, vol. 2, pp. 35-55, 2012.

10. C. Ronse, "Erosion of narrow image features by combination of local low rank and max filters," in Image Processing and its Applications, Proceedings 2nd IEE International Conference on, 1986, pp. $77-81$.

11. C. Ronse and H. Heijmans, "The algebraic basis of mathematical morphology : II. openings and closings," Computer Vision, Graphics, and Image Processing, vol. 54, no. 1, pp. 74-97, 1991.

12. H. Heijmans, "Morphological image operators," Advances in Electronics and Electron Physics Suppl., Boston: Academic Press, vol. 1, 1994.

13. P. Dokládal and E. Dokládalová, "Computationally efficient, one-pass algorithm for morphological filters," Journal of Visual Communication and Image Representation, vol. 22, pp. 411-420, 2011.

14. S. Perreault and P. Hébert, "Median filtering in constant time," IEEE Transactions on Image Processing, vol. 16, no. 9, pp. 2389-2394, September 2007. 